\title{
Localized gap modes in nonlinear dimerized Lieb lattices
}

\author{
P. P. Beličev, ${ }^{1}$ G. Gligorić, ${ }^{1}$ A. Maluckov, ${ }^{1}$ M. Stepić, ${ }^{1}$ and M. Johansson ${ }^{2}$ \\ ${ }^{1} P^{*}$ Group, Vinča Institute of Nuclear Sciences, University of Belgrade, P.O. Box 522, 11001 Belgrade, Serbia \\ ${ }^{2}$ Department of Physics, Chemistry and Biology, Linköping University, SE-581 83 Linköping, Sweden
}

(Received 30 October 2017; published 28 December 2017)

\begin{abstract}
Compact localized modes of ring type exist in many two-dimensional lattices with a flat linear band, such as the Lieb lattice. The uniform Lieb lattice is gapless, but gaps surrounding the flat band can be induced by various types of bond alternations (dimerizations) without destroying the compact linear eigenmodes. Here, we investigate the conditions under which such diffractionless modes can be formed and propagated also in the presence of a cubic on-site (Kerr) nonlinearity. For the simplest type of dimerization with a three-site unit cell, nonlinearity destroys the exact compactness, but strongly localized modes with frequencies inside the gap are still found to propagate stably for certain regimes of system parameters. By contrast, introducing a dimerization with a 12-site unit cell, compact (diffractionless) gap modes are found to exist as exact nonlinear solutions in continuation of flat band linear eigenmodes. These modes appear to be generally weakly unstable, but dynamical simulations show parameter regimes where localization would persist for propagation lengths much larger than the size of typical experimental waveguide array configurations. Our findings represent an attempt to realize conditions for full control of light propagation in photonic environments.
\end{abstract}

DOI: 10.1103/PhysRevA.96.063838

\section{INTRODUCTION}

Photonic lattices (PLs) are well known periodically modulated optical materials which exhibit a band-gap structure with allowed and forbidden bands. The latter ones are also called "gaps" since there is no possibility for extended Bloch modes to be guided on frequencies from forbidden bands [1]. However, the light at these "lost" frequencies can be guided if there are some defects, inhomogeneities or disorder within the system, or in the presence of nonlinearity. In all these cases trapping of light is observed owing to the phenomena based either on Anderson localization [2], defect modes [3], or modulation instability [4].

Localized structures of the solitary type such as discrete solitons, gap solitons, and breathers have been identified in the PLs [5]. Moreover, nonuniform PLs present different kinds of superlattice structures [6] where additional periodicity opens an extra minigap in the energy spectrum preventing linear light propagation through the system, but providing nonlinear trapping of light as another way of light guiding in the form of a gap soliton. Gap solitons were predicted and experimentally realized in binary PLs with alternating channel widths but constant separations between channels [7,8] and in PLs with constant widths of waveguides but alternating separations between them in the presence of cubic type nonlinearities $[9,10]$.

With developed fabrication technologies it is possible to fabricate tailor-made photonic structures with optimized properties enabling a plethora of interesting phenomena related to light confinement [11]. One of them is localization of light as a consequence of the specific topology of the waveguide distribution and, for example, photonic topological insulators were observed [12,13]. Moreover, for particular geometries, conditions necessary for destructive wave interference can be achieved even in periodic PLs, without the presence of disorder, defects, or nonlinearity. Such systems are known as flat band systems, due to their energy spectra containing a perfectly flat energy band that provides diffractionless light propagation. Seminal papers showed that interesting effects in condensed matter systems [14-16] are observed. Additionally, the influence of the singularity of flat band systems was investigated in the frame of the fractional quantum and magnon Hall effect $[17,18]$ and energy localization in the presence of magnetic fields [19], spin-orbit coupling [20,21], or disorder $[22,23]$. Recently this topic attracted a lot of attention. Light localization effects in 1D nonlinear kagome and ladder ribbons (uniform and binary) [24,25], as well as in 2D kagome lattices [26-29] were investigated.

One of the simplest two-dimensional (2D) flat band geometries is the Lieb lattice. Geometrically, this system resembles the ordinary square lattice with one site missing on every even step of the lattice grid [30]. The absence of these sites changes the energy spectrum giving rise to a completely flat, nondiffracting band surrounded by two dispersive bands, touching them in Dirac points within the Brillouin zone. Results on theoretical predictions and experimental confirmation of light propagation without spreading have been published in Refs. [30-33]. However, the presence of Kerr nonlinearity in the system may exhibit conical diffraction at the Dirac cone $[30,34]$. Due to their simple geometry, Lieb lattices are in the focus of research in ultracold systems as an optical trap for fermions [35], providing existence of magnon Hall effect in spite of the presence of inversion symmetry [18], protection and formation of robust zero modes localized at point defects [36], optimization of the BCS critical temperature and superfluid weight [37], and lifting the flat band modes by PT-symmetric perturbation due to thresholdless PT-symmetry breaking [38]. Moreover, it has been shown that the Lieb lattice acts as a good platform for analyzing various topological transitions in Chern insulators [20,39-41].

As in every flat band system, eigenfunctions belonging to the Lieb lattice flat band are entirely degenerate states. Any superposition of these states is nondiffracting as well. The fundamental flat band eigenmode in this model can be chosen as a four-site closed ring with staggered phase relation between neighboring sites in the ring. In general, it has been 
shown theoretically and demonstrated experimentally that linear compact localized modes of the ring type can be formed within Lieb systems [30-33].

In this paper we investigate, both theoretically and numerically, the existence and stability of linear and nonlinear localized modes in the neighborhood of the corresponding linear flat band in 2D dimerized Lieb lattices, exhibiting Kerr type of nonlinearity. Our interest is mainly on modes created inside the gap in the eigenvalue spectra opened due to the dimerizations. It has recently been shown [42] that for the uniform, gapless, Lieb lattice, any weak nonlinearity will destabilize the compact ring modes, while restabilization occurs only in the large-nonlinearity regime. Following the model presented in Ref. [30] with on-site nonlinearity, we examine the existence and behavior of compact localized modes of closed-ring shape for two types of dimerizations of the nonlinear Lieb system. For the simplest type of dimerization with a three-site unit cell, which has been studied earlier in other theoretical contexts $[20,37,39,40]$ and also recently realized experimentally with microwave resonators [36], we prove that, although the compact four-site ring modes of the uniform Lieb lattice still persist in the linear limit, their amplitude distribution becomes nonhomogeneous which prohibits their existence as exact stationary solutions in the presence of nonlinearity. Instead, we found numerically that strongly localized gap modes with nonzero tails can exist in the presence of nonlinearity. Their existence range increases for stronger dimerization. These modes are shown to be generally stable for small amplitudes and typically develop long-lived "breathing" oscillations when perturbed. Then we introduce another type of dimerization of the Lieb lattice, with a 12-site unit cell and a fourfold degenerate flat band. For this system, we prove that for every second four-site ring mode the amplitude distribution will remain homogeneous, and thus these modes will persist also for the nonlinear model.
However, numerical investigations indicate that these compact modes are generally unstable, although the instabilities in certain parameter regimes are weak and possibly not detectable with present experimental setups for optical waveguide arrays.

\section{MODEL EQUATIONS}

The uniform 2D Lieb lattice [30-33] pattern is schematically presented in Fig. 1(a), if all coupling constants $\left(V_{x 1}, V_{x 2}, V_{y 1}\right.$, and $\left.V_{y 2}\right)$ are taken to be identical. Its primitive cell contains three sites as denoted by the dashed square in the figure. By spatial translation of this fundamental block in horizontal and vertical directions, a Lieb lattice with flat band energy spectrum is obtained. In general, however, couplings between neighboring sites can take arbitrary (even random) values and still keep the particular property of the Lieb lattice with a flat band in the linear spectrum [32].

In the following, we will consider two particular types of dimerized Lieb lattices. The first one, here denoted as "type I" and described in several earlier works $[20,36,37,39,40]$, can be realized by arranging the lattice pattern according to the scheme in Fig. 1(b). The primitive cell contains the same three sites (bounded in the figure with a dashed square) as in the uniform case. Notice that, while the uniform Lieb lattice exhibits reflection symmetry through diagonal as well as antidiagonal axes through all four-bonded $(b)$ sites, the "type I" dimerized lattice, having different intracell and intercell couplings, loses the antidiagonal symmetry. This will be shown to be important for the possibility of existence of compact modes for the nonlinear model below.

For the case of general periodic Lieb lattice with three-site unit cell and four different coupling constants [Fig. 1(a)], the evolution of the light wave amplitude through the system can be modeled by the following set of dimensionless
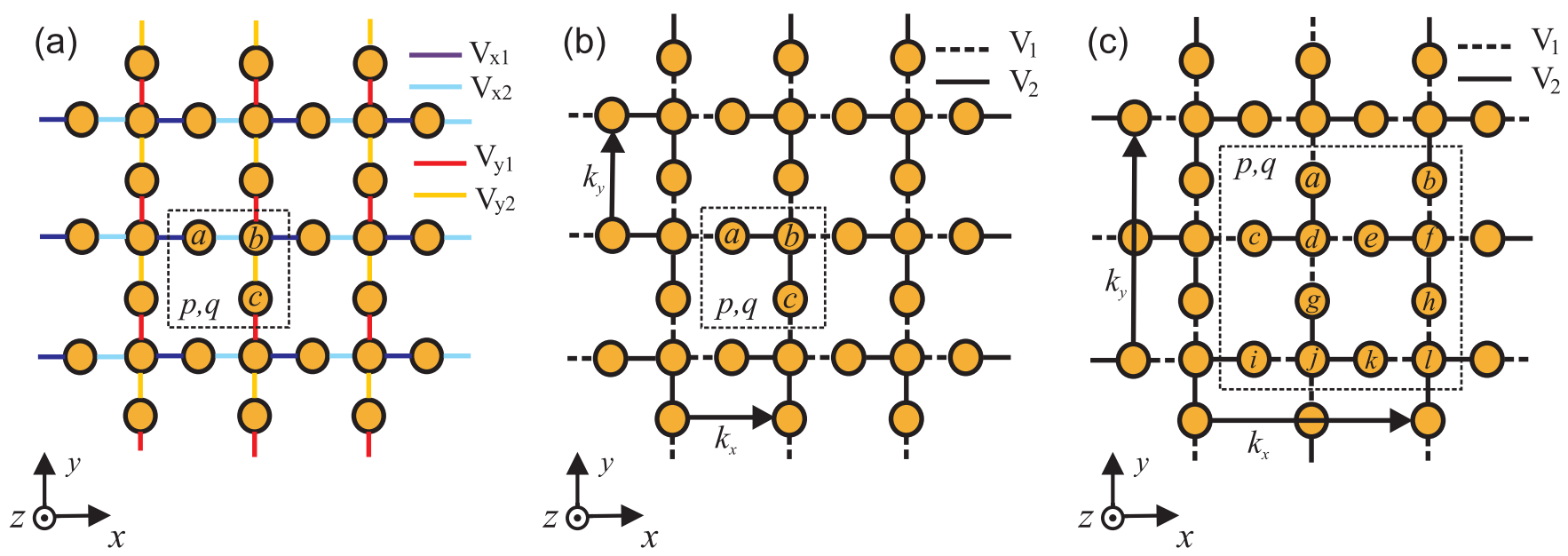

FIG. 1. Schematic representations of two classes of dimerized Lieb lattices: (a) three-site unit cell with general coupling coefficients; (b) special case of (a) with a diagonal symmetry axis through $b$ sites ("type I"); (c) 12-site unit cell ("type II") with diagonal symmetry axis through sites $f$ and $j$, and antidiagonal symmetry axis through sites $d$ and $l$. The primitive cells are denoted by dashed squares. Amplitudes within the characteristic sites are denoted by $a, b, c, d, e, f, g, h, i, j, k$, and $l$, respectively. Here, $p(q)$ denotes cell index in the $x$ ( $y$ ) direction, where $p, q \in[1, N]$. The Bloch vector corresponding to translations between two neighboring fundamental blocks is described by $k_{x}$ in the horizontal and $k_{y}$ in the vertical direction, respectively. Different colors of lines in (a) represent different strengths of coupling between sites, while solid and dashed lines stand for the two coupling constants in (b) and (c). 
differential-difference nonlinear Schrödinger equations:

$$
\begin{aligned}
& \mathrm{i} \partial_{z} a_{p, q}+V_{x 1} b_{p-1, q}+V_{x 2} b_{p, q}+\gamma\left|a_{p, q}\right|^{2} a_{p, q}=0 \\
& \mathrm{i} \partial_{z} b_{p, q}+V_{x 2} a_{p, q}+V_{x 1} a_{p+1, q}+V_{y 2} c_{p, q}+V_{y 1} c_{p, q+1} \\
& \quad+\gamma\left|b_{p, q}\right|^{2} b_{p, q}=0 \\
& \mathrm{i} \partial_{z} c_{p, q}+V_{y 2} b_{p, q}+V_{y 1} b_{p, q-1}+\gamma\left|c_{p, q}\right|^{2} c_{p, q}=0
\end{aligned}
$$

where $a_{p, q}, b_{p, q}$, and $c_{p, q}$ are mode amplitudes at $a, b$, and $c$ sites, respectively. Coupling constants along the $x$ direction are denoted by $V_{x 1}$ and $V_{x 2}$, along the $y$ direction by $V_{y 1}$ and $V_{y 2}$, while $z$ stands for the propagation coordinate. The effective (normalized) nonlinear coefficient $\gamma$ may represent focusing $(\gamma=1)$ or defocusing $(\gamma=-1)$ types of Kerr nonlinearity. Conserved quantities in the system are norm (power), $P$ :

$$
P=\sum_{p, q}\left(\left|a_{p, q}\right|^{2}+\left|b_{p, q}\right|^{2}+\left|c_{p, q}\right|^{2}\right),
$$

and Hamiltonian:

$$
\begin{aligned}
H= & -\frac{1}{2} \sum_{p, q}\left\{\gamma\left(\left|a_{p, q}\right|^{4}+\left|b_{p, q}\right|^{4}+\left|c_{p, q}\right|^{4}\right)\right. \\
& +\left[a_{p, q}\left(V_{x 1} b_{p-1, q}+V_{x 2} b_{p, q}\right)^{*}\right. \\
& +b_{p, q}\left(V_{x 2} a_{p, q}+V_{x 1} a_{p+1, q}+V_{y 2} c_{p, q}+V_{y 1} c_{p, q+1}\right)^{*} \\
& \left.\left.+c_{p, q}\left(V_{y 2} b_{p, q}+V_{y 1} b_{p, q-1}\right)^{*}+\text { c.c. }\right]\right\}
\end{aligned}
$$

In addition, we construct another type of dimerized Lieb lattice with a primitive cell containing twelve sites, as denoted by the dashed square in Fig. 1(c). This lattice will be noted in the following as the "type II" dimerized Lieb lattice. In contrast to the type I dimerization in Fig. 1(b), this lattice has bond alternation of vertical couplings [ $V_{y 1}$ and $V_{y 2}$ in Fig. 1(a)] also along the horizontal $(x)$ direction, and bond alternation of horizontal couplings [ $V_{x 1}$ and $V_{x 2}$ in Fig. 1(a)] also along the vertical $(y)$ direction. With this construction, one regains the antidiagonal reflection symmetry axes through every second four-bonded site [sites $d$ and $l$ in Fig. 1(c)], at the price of losing the diagonal symmetry axes around these sites.

The corresponding set of model equations is completely analogous to Eq. (1) but algebraically too cumbersome for writing out explicitly here. It contains 12 equations for complex mode amplitudes which are coupled as schematically shown in Fig. 1(c). As will be shown in the following, the symmetry of this particular type of dimerized Lieb lattice allows for the possibility to bond ring-compact localized modes with uniform amplitude, analogous to rings in the uniform Lieb geometry. This system is also characterized by two invariants, power and Hamiltonian, with definitions analogous to Eqs. (2) and (3), respectively.

\section{Linear dimerized Lieb lattices}

We consider first the Lieb dimerization with three-site unit cell and general coupling coefficients [Fig. 1(a)]. By introducing a stationary solution, $\left\{a_{p, q}, b_{p, q}, c_{p, q}\right\} \sim e^{\mathrm{i}\left(k_{x} p+k_{y} q+\beta z\right)}$, with the corresponding 2D Bloch wave vector $\vec{k}=\left(k_{x}, k_{y}\right)$, into the linear part of Eq. (1), we derive the dispersion relation whose solutions can be written in the form:

$$
\beta_{0}=0, \quad \beta_{1,2}= \pm \sqrt{V_{x 1}^{2}+V_{x 2}^{2}+V_{y 1}^{2}+V_{y 2}^{2}+2 V_{x 1} V_{x 2} \cos k_{x}+2 V_{y 1} V_{y 2} \cos k_{y}}
$$

We distinguish three linear bands, two of which are dispersive and one is completely flat: $\beta_{0}=0$. Highly confined states that belong to the flat band are the so-called "ring" solutions which have four peaks with alternating sign of amplitude. In the uniform lattice these rings are compact modes characterized by equal absolute values of the peak amplitudes. In the case of the dimerized lattices in Fig. 1(a) with arbitrary couplings among sites, it is possible to construct a minimal ring mode at $\beta_{0}=0$ if the following relations are obeyed:

$$
\begin{aligned}
a_{p, q} & =-\frac{V_{y 2}}{V_{x 2}} c_{p, q}, \quad a_{p, q-1}=-\frac{V_{y 1}}{V_{x 2}} c_{p, q}, \\
c_{p-1, q} & =\frac{V_{x 1}}{V_{x 2}} c_{p, q} .
\end{aligned}
$$

Any linear combination of ring modes is an eigensolution of the system.

Specifically, we focus on the "simplest" special case of the type I dimerization [Fig. 1(b)], setting the coupling constants to fulfill the following dependence: $V_{x 1}=V_{y 1}=V_{1}$ (intercell coupling) and $V_{x 2}=V_{y 2}=V_{2}$ (intracell coupling). In this case, the dispersion relation (4) reduces to the form given in Ref. [37]. An example of a fundamental ring mode and its linear combinations in this linear dimerized Lieb system are represented in Figs. 2(a)-2(c). Due to the symmetry properties of the introduced dimerization, the profiles of the fundamental ring modes [Fig. 2(a)] are antisymmetric under diagonal reflection through the ring center but asymmetrical under antidiagonal reflection, as dictated by Eq. (5).

Depending on the symmetry of the introduced dimerization, ring modes with profiles of different symmetry can be achieved in the lattice. In the case of the type II Lieb lattice dimerization, linearization of the set of model equations analogous to (1) will lead to the dispersion relation (eigenenergy spectrum):

$$
\begin{aligned}
\beta_{0} & =0 \\
\beta_{1,2} & = \pm \sqrt{2} \sqrt{V_{1}^{2}+V_{2}^{2}-V_{1} V_{2}\left(\cos k_{x} / 2+\cos k_{y} / 2\right)}, \\
\beta_{3,4} & = \pm \sqrt{2} \sqrt{V_{1}^{2}+V_{2}^{2}+V_{1} V_{2}\left(\cos k_{x} / 2-\cos k_{y} / 2\right)}, \\
\beta_{5,6} & = \pm \sqrt{2} \sqrt{V_{1}^{2}+V_{2}^{2}+V_{1} V_{2}\left(-\cos k_{x} / 2+\cos k_{y} / 2\right)}, \\
\beta_{7,8} & = \pm \sqrt{2} \sqrt{V_{1}^{2}+V_{2}^{2}+V_{1} V_{2}\left(\cos k_{x} / 2+\cos k_{y} / 2\right)} .
\end{aligned}
$$

Figure 3 depicts band structures obtained for uniform lattice (a), and dimerized lattices of type I (b) and type II (c), respectively. In the case of the uniform Lieb lattice, the flat band touches the dispersive bands in points at the end of the Brillouin zone. By introducing additional periodicity in the system either 

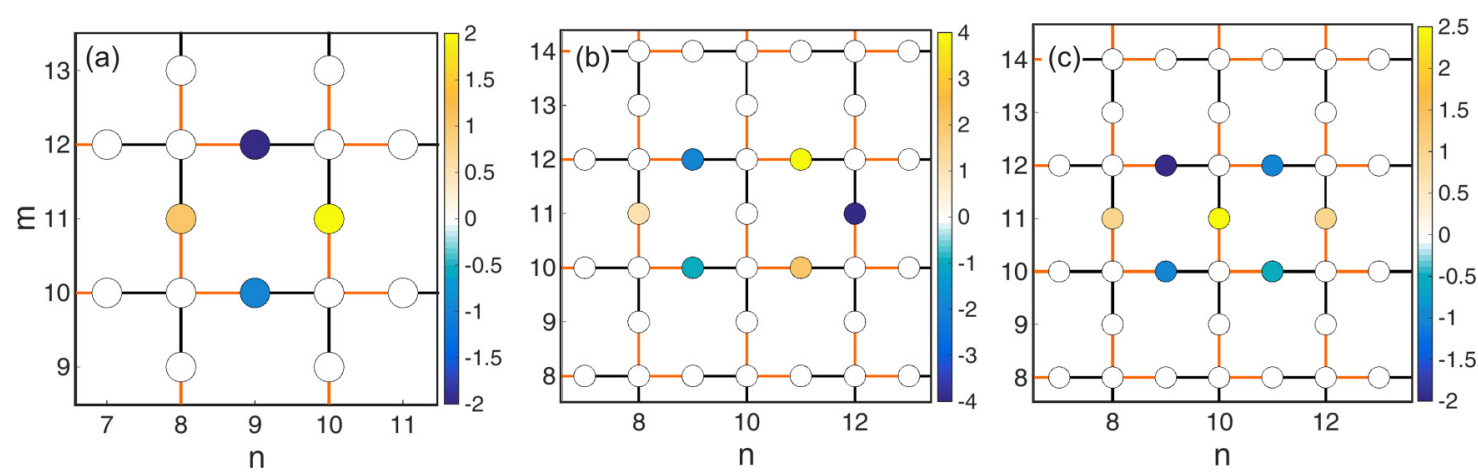

FIG. 2. Real parts of amplitude for: (a) minimal 4-peak ring mode; (b) 6-peak ring mode-superposition of two "out-of-phase" 4-peak modes that cancel each other at the central $c$ site; (c) 7-peak ring mode—superposition of two "in-phase" 4-peak modes. The amplitude changes sign from site to site, according to the relation (5). Couplings $V_{x 1}=V_{y 1}=0.5$ are marked by orange (gray) lines, while $V_{x 2}=V_{y 2}=1$ are marked by black ones. The indices $m$ and $n$ represent the site numbers of the lattice.

of type I or II, gaps (minigaps in the following) open symmetrically above and below the flat band as can be seen in Figs. 3(b) and 3(c), respectively. This may be contrasted with the case of the Lieb lattice with constant couplings but binary modulated on-site energies, where a gap appears only on one side of the flat band [43]. The size of the primitive cell dictates the complexity of the energy spectrum. For the type I dimerization we distinguish three bands, while the type II apparently provides the existence of nine distinct linear bands. In both cases, only one band is completely flat and degenerated $(\beta=0)$, Figs. 3(b) and 3(c). The widths of the minigaps depend on the ratio of coupling constants $V_{1} / V_{2}$ as depicted in Fig. 3(d). As follows from (4) and (6), the gap widths $\Delta$ are in both cases given by:

$$
\Delta=\sqrt{2}\left|V_{2}-V_{1}\right|
$$

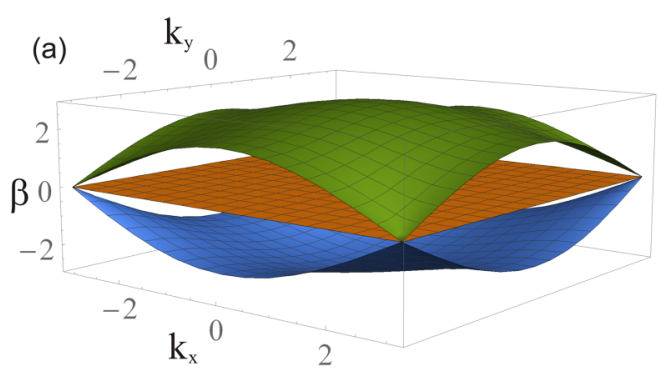

(c)

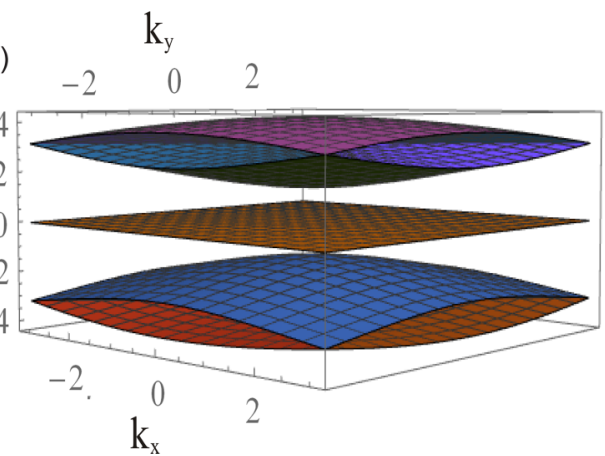

so that for increasing dimerization the minigaps become wider, directly proportional to the difference between the coupling constants. Moreover, the flat band is preserved for every ratio of the coupling constants. In order to preserve stronger coupling between sites within a primitive cell $(p, q)$ [as shown in Figs. 1(b) and 1(c)], we always set coupling constant $V_{2}$ to have a higher value than $V_{1}$.

The fact that the type II dimerization, with a 12-site unit cell, only shows nine distinct bands is explained by the flat band itself being fourfold degenerate, since one may construct four different fundamental compact four-site ring modes involving nonequivalent sites from a given unit cell [e.g., with the labeling of Fig. 1(c), the modes contain sites $\{e, g, h, k\},\{a, c\},\{b\}$, and $\{i\}$, respectively, from cell $(p, q)$, with the remaining sites belonging to neighboring cells]. Due

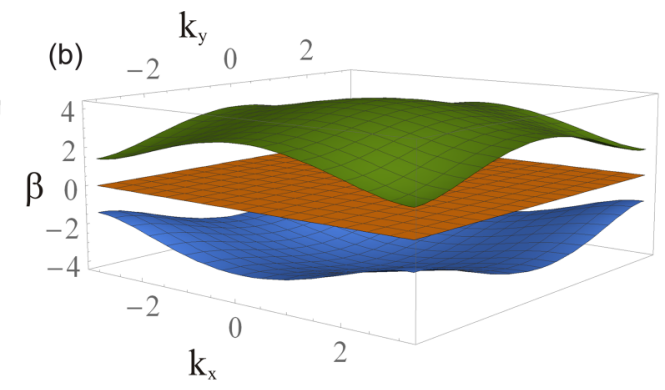

(d)

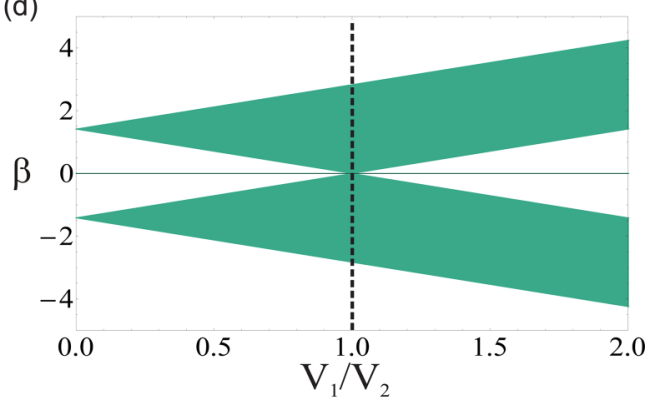

FIG. 3. Dispersion relations (energy spectra) for the cases of (a) uniform Lieb lattice, and dimerized Lieb lattices of (b) type I, and (c) type II, when $V_{1}=1$ and $V_{2}=2$. Plot (d) shows influence of strength of dimerization $V_{1} / V_{2}$ on gap width of both dimerized Lieb lattices of I and II type. Second coupling parameter $V_{2}$ is fixed to one. Dashed vertical line marks the case of uniform lattice for which only semi-infinite gaps exist. 


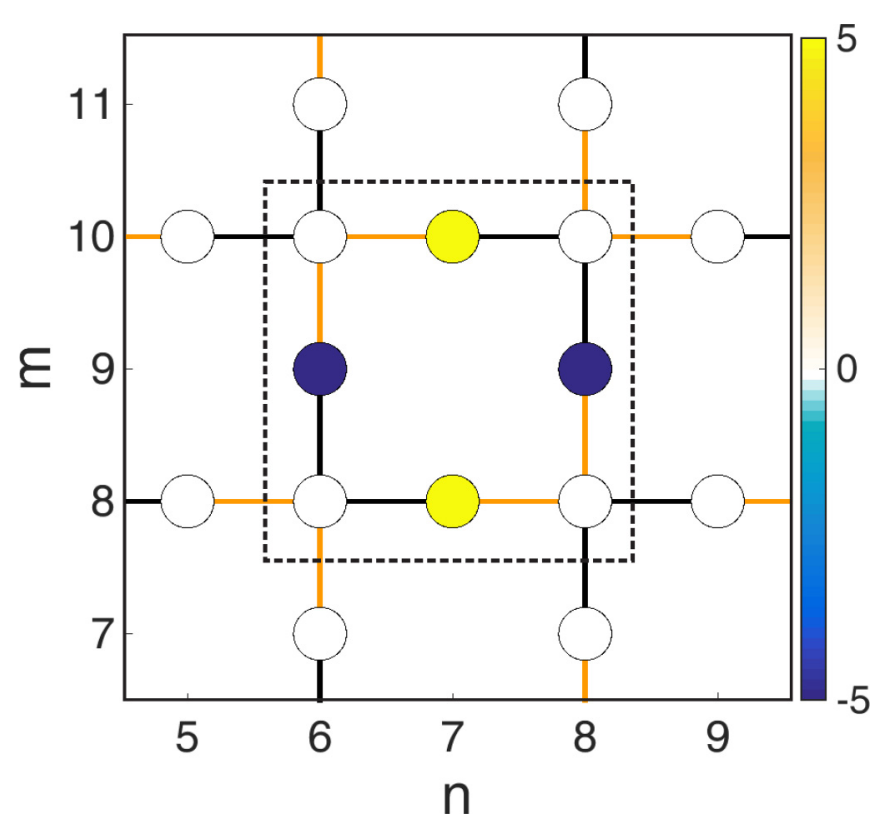

FIG. 4. The amplitude distribution of a 4-ring mode in the dimerized Lieb lattice of type II. Orange (gray) and black solid lines depict coupling strength $V_{1}$ and $V_{2}$, respectively. The dashed square frames a block with the particular couplings between sites that provides existence of ring modes.

to the particular symmetries of the type II dimerized Lieb lattice, these highly confined states that belong to the flat band will be antisymmetric under diagonal as well as antidiagonal reflections with respect to their ring centers, and thus they will have four peaks with alternating sign of amplitudes and equal absolute values (see Fig. 4). In other words, the fundamental flat band eigenmodes will have profiles of the same symmetry as those obtained for a uniform lattice.

Note also that in the "anticontinuum" limit, with one of the two coupling constants vanishing (e.g., $V_{1}=0$ ), both configurations (type I and type II) transform to a system of mutually uncoupled trimers with a $90^{\circ}$ angle at the central site. Each trimer is actually a fundamental block that supports the existence of a highly localized mode with amplitude distribution $\{a, b, c\}=\{1,0,-1\}$ (type I lattice), and $\{e, f, h\}=$ $\{1,0,-1\}$ or $\{g, j, k\}=\{1,0,-1\}$ (type II lattice), respectively. For the type I dimerization [Fig. 1(b)], these trimers all point in the same directions, and thus they cannot simply be combined to form a four-site ring mode. On the other hand, for the type II dimerization [Fig. 1(c)] the trimers will be "pointing" alternately upwards and downwards when moving in the horizontal direction, and thus any four-site ring mode may be viewed as the combination of two diagonally located "anticontinuous" trimer modes.

\section{NONLINEAR DIMERIZED LIEB LATTICES}

The influence of nonlinearity in dimerized ("binary") flat band systems have previously been studied for 1D kagome and ladder strips [25]. There, three types of nonlinear ring solutions were found to exist: unstaggered, staggered, and vortex. The binarism in strips provided formation of closed six/four site structures mutually coupled via the same coupling constants. In this way, conditions for generation of stable ring solutions were ensured in the nonlinear regime, too. However, as may be deduced from the previous section, the additional periodicity in the type I Lieb dimerized lattice does not allow for constructing an arrangement of sites mutually coupled with the same strength into ring formation, in contrast to the case of the type II dimerization. This will be shown more explicitly in the following.

Below we study the regime when nonlinearity of Kerr type is "turned on" in dimerized Lieb lattices of type I (Sec. III A) and II (Sec. III B). Due to the symmetries of the linear energy spectra (4), (6) of both systems with respect to $\beta=0$, equivalent results are obtained for defocusing and focusing types of nonlinearity in (1). In the case of defocusing nonlinearity localized modes will be generated in the gap below the flat band, while in the case of focusing nonlinearity, light localization will be possible in the gap above the flat band. Due to this fact, we consider only the case of focusing type of nonlinearity $(\gamma=1)$, without loss of generality.

The stability of the nonlinear localized modes obtained below is studied applying the linear stability analysis (LSA) and direct dynamical simulations. Both procedures are performed by introducing small perturbations to the modes. Briefly, the LSA reduces to the derivation of the eigenvalues $(\mathrm{EVs})$ of the characteristic matrix of the linearized equations for small perturbations. The instability is then related to the nonzero real part of the corresponding EVs, which are actually the growth rates of the perturbations [44-46]. Two types of instability can be distinguished, the exponential (purely real EV) and oscillatory (complex EV) type. On the other hand, the dynamical stability of a certain mode is confirmed by observing the effect of a small random amplitude perturbation initially added to the mode. The perturbation is numerically modeled by applying a uniform random number generator, which gives a set of random numbers from the interval $[-0.01,0.01][46]$.

\section{A. Type I dimerization: Gap solitary modes}

For the type I dimerization, using an ansatz in the form of a stationary solution $\left\{a_{p, q}(z), b_{p, q}(z), c_{p, q}(z)\right\}=$ $\left\{A_{p, q}, B_{p, q}, C_{p, q}\right\} e^{\mathrm{i} \beta z}$, with $z$-independent amplitudes $\left\{A_{p, q}, B_{p, q}, C_{p, q}\right\}$, and imposing the amplitude conditions (5) for a fundamental four-site compact ring, we obtain from (1) the following relations for the nonlinear frequency shift $\beta$ :

$$
\begin{aligned}
A_{p, q} & =-C_{p, q} \rightarrow \beta=\gamma\left|C_{p, q}\right|^{2}, \\
A_{p, q-1} & =-\frac{V_{1}}{V_{2}} C_{p, q} \rightarrow \beta=\gamma\left(\frac{V_{1}}{V_{2}}\right)^{2}\left|C_{p, q}\right|^{2}, \\
C_{p-1, q} & =-\frac{V_{1}}{V_{2}} A_{p, q}=\frac{V_{1}}{V_{2}} C_{p, q} \rightarrow \beta=\gamma\left(\frac{V_{1}}{V_{2}}\right)^{2}\left|C_{p, q}\right|^{2} .
\end{aligned}
$$

Thus, unless $\gamma=0$ (linear model) or $V_{2}=V_{1}$ (uniform Lieb lattice) we see that sites located on opposite sides of the antidiagonal through the center of the ring mode would get different nonlinear frequency shifts, violating the assumption of stationarity, and thus the compact ring structure cannot be preserved in the nonlinear regime.

Still, we would expect that, at least as long as the nonlinear frequency shift $\beta$ is small (i.e., for small amplitudes or weak 

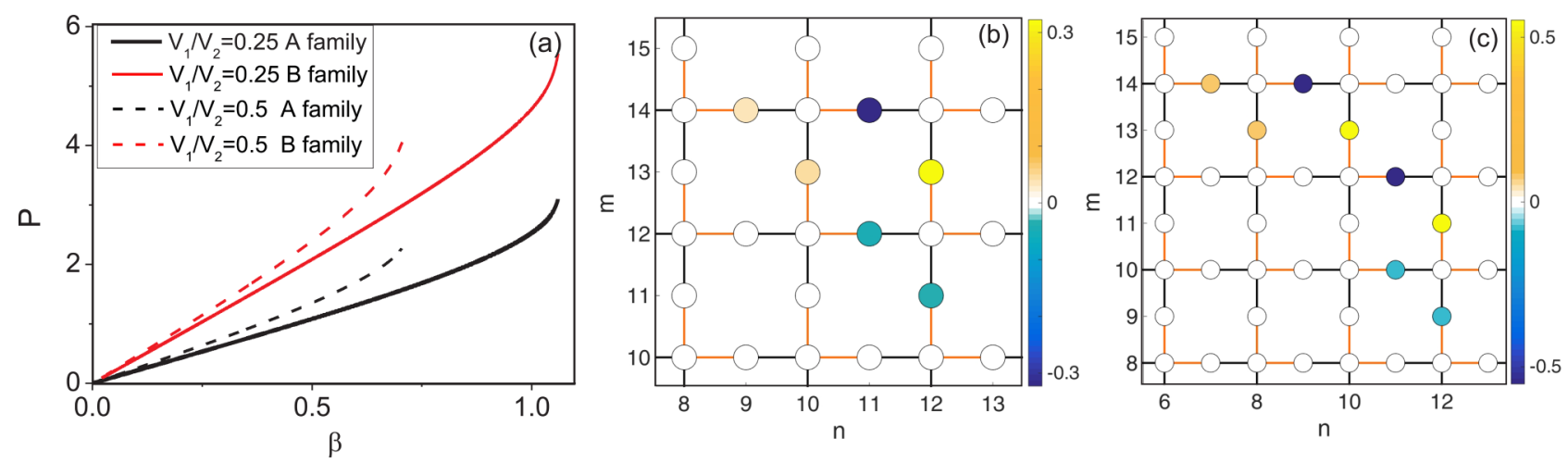

FIG. 5. Nonlinear localized modes in the minigap of type I dimerized Lieb lattice: (a) Power vs propagation constant for two families ( $A$ and $B$ ) and corresponding amplitude profiles of a typical small-amplitude mode from (b) family $A(\beta=0.101)$ and (c) family $B(\beta=0.3)$ when $V_{1} / V_{2}=0.25$. Orange (gray) solid lines stand for $V_{1}=0.25$ coupling strength, while black ones represent $V_{2}$ coupling. The $m$ and $n$ represent the site numbers of the lattice. Power curves in (a) show the existence range of solutions until the upper gap edge, located at $\beta=0.75 \sqrt{2} \approx 1.06$ (solid curves, $V_{1}=0.25$ ) and $\beta=0.5 \sqrt{2} \approx 0.71$ (dashed curves, $V_{1}=0.5$ ), respectively.

nonlinearities), there should exist some family of (generally noncompact) stationary solutions which connects to the exact compact modes in the limit of the uniform Lieb lattice, $V_{1} / V_{2} \rightarrow 1$.

As $V_{1} / V_{2}$ departs from 1 , energy injected locally in the system cannot equally distribute among the four ring sites. Instead, the numerical simulations presented below show that it primarily spreads to $a$ and $c$ sites of cells which are closest to the ring block. This will lead to the formation of a localized mode consisting of the four ring sites and two additional neighboring sites where light (energy) has been redistributed.

To search for such families of gap solitary modes as exact stationary solutions to Eq. (1), we implement a standard numerical continuation procedure based on the modified Powell minimization method [47], where exact four-site compact modes of the linear model may be used as initial trial solutions. As a typical criterion for identifying numerically exact solutions, a convergence tolerance of $10^{-8}$ is used. In Fig. 5, we illustrate properties of two different families of nonlinear stationary modes, denoted as $A$ and $B$, found to exist in the minigap of the type I dimerized lattice. Family $A$ [Fig. 5(b)] keeps ring's symmetry properties of having its main excitation on the four ring sites [cf. Fig. 2(a)] and being antisymmetric around the diagonal through the ring center, but delocalizing mainly to the sites strongly coupled to the two antidiagonal $b$ sites connecting the ring. On the other hand, family $B$ [Fig. 5(c)] can be viewed as a nonlinear superposition of two $A$ modes connected antidiagonally at $b$ site $[(m, n)=(12,10)$ in Fig. $5(\mathrm{c})]$, with phases such that the whole mode is antisymmetric around the diagonal through this site. Note also from Fig. 5(a) that the power carried by a solution from family $B$ is approximately twice as big as that of a solution from family $A$ for the same $\beta$. Both mode families are characterized by strong localization in the vicinity of the flat band (small $\beta$ ), while they become broader as $\beta$ increases and they get closer to the edge of the upper dispersive band given by Eq. (7). Approaching the dispersive band (higher $\beta$ ), very small amounts of energy can be localized also in $b$ sites.

For stronger dimerization, the localization of light gets more pronounced on sites coupled by the higher coupling constant, which enables formation of highly peaked modes.
Figure 6 depicts "compactness" as a function of $V_{1} / V_{2}$. Compactness indicates ratio of the power of localized mode (on 4 and 2 main sites) with respect to total power launched in the system. Red (upper) solid line depicts this ratio for the four main sites in the case of ring structure (Compactness $=$ $\left.\left(\left|a_{14,11}\right|^{2}+\left|a_{12,11}\right|^{2}+\left|c_{13,12}\right|^{2}+\left|c_{13,10}\right|^{2}\right) / P\right)$, while the blue (lower) one indicates compactness for two main sites linked by stronger coupling thus forming a trimer in the limit $V_{1} / V_{2} \rightarrow 0$ $\left(\right.$ Compactness $\left.=\left(\left|a_{14,11}\right|^{2}+\left|c_{13,12}\right|^{2}\right) / P\right)$. Here, site indexing $(m, n)$ corresponds to the one presented in Fig. 5 .

In the case of family $A$ solutions, blue (lower) and red (upper) lines approach each other with the decrease of ratio $V_{1} / V_{2}$ [see Fig. 6(a)]. In the limit $V_{1} \ll V_{2}$, when the lattice basically reduces to weakly coupled trimers as pointed out in Sec. II A, the family $A$ reduces to a trivial nonlinear two-site antisymmetric solution localized only at sites $a$ and $c$ within a single trimer, while the family $B$ (not shown in Fig. 6) reduces to the superposition of two such identical solutions positioned in two neighboring trimers along the antidiagonal. In fact, solutions continued from these trimer modes exist not only in the gap but also above the linear spectrum, but their shapes bear no resemblance to flat band modes in this regime. On the other hand, as the coupling ratio approaches the case of uniform lattice, the energy of the gap soliton slowly spreads to the neighboring sites of the two dominant sites within the ring formation (see mode distribution for $V_{1} / V_{2}=$ 0.9 in Fig. 6). With further decrease of dimerization strength this mode profile doesn't change and "survives" in the case of uniform lattice as a nonlinear stationary solution carrying around $93 \%$ of the total amount of power on four ring sites. Thus, there is no continuation of solutions of family $A$ to the exact compact modes in the limit of the uniform Lieb lattice, $V_{1} / V_{2} \rightarrow 1$.

Beside $A$ and $B$ families of nonlinear gap solutions, we found a narrow region of existence of another class of nonlinear localized solution which connects to the exact compact modes in the limit of the uniform Lieb lattice as depicted in Fig. 6(b). This class of solutions does not preserve full compactness of the nonlinear ring mode when continued away from the uniform case. Although its power is mostly localized in the ring formation, a small amount 

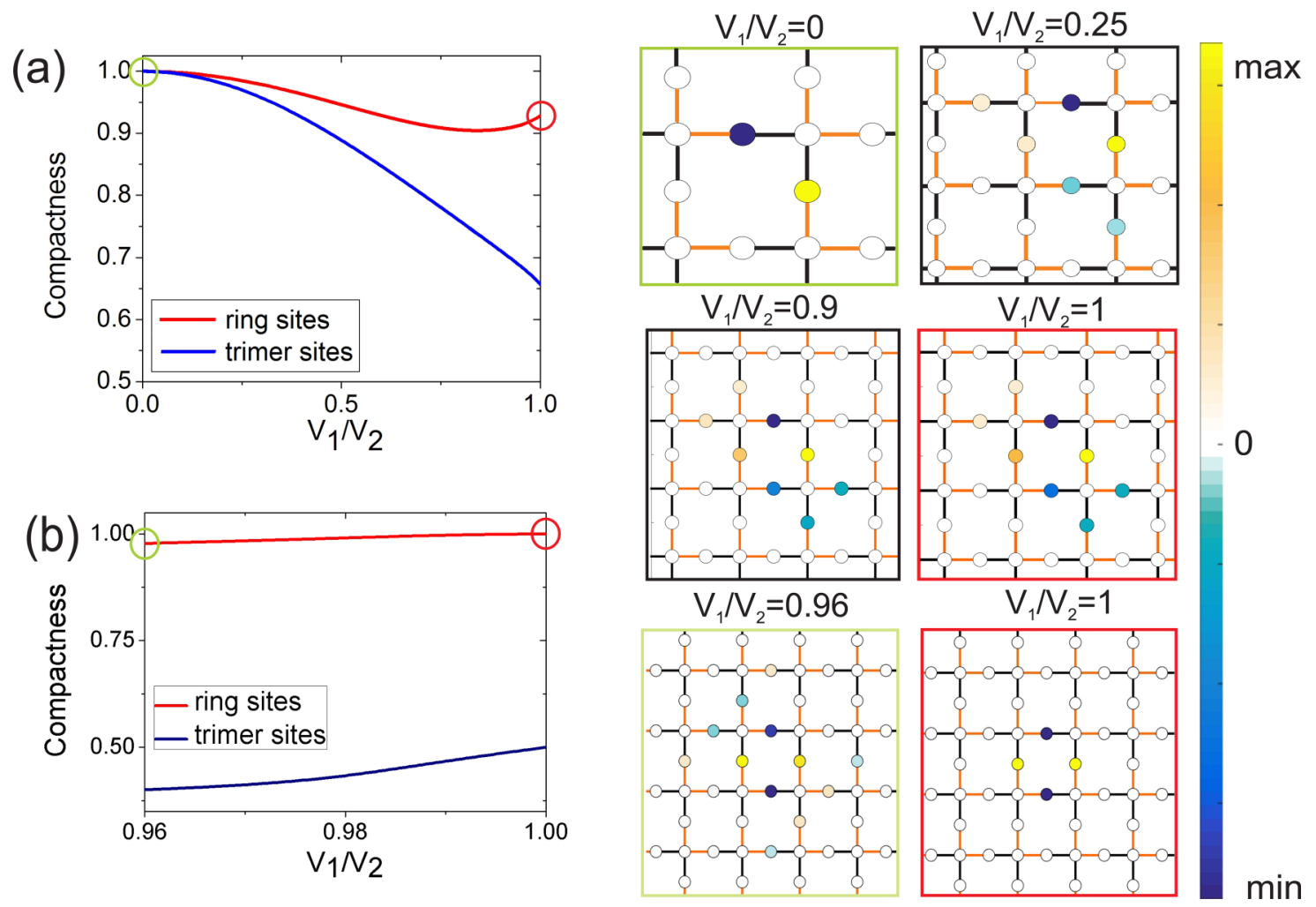

FIG. 6. Compactness vs $V_{1} / V_{2}$ with $V_{1}<1$ and $V_{2}=1$ for (a) family $A$ and (b) nonlinear ring solitons, characterized with $\beta=0.001$. The upper (red) lines show compactness on all four ring sites, while the lower (blue) lines show compactness on the two ring sites belonging to a strongly bonded trimer. Panels on the right show amplitude distributions of modes for different coupling strengths: trimer solution $\left(V_{1} / V_{2}=0\right)$ and gap soliton of family $A$ for $V_{1} / V_{2}=0.25$-first row, and gap solitons of family $A$ for $V_{1} / V_{2}=0.9$ and $V_{1} / V_{2}=1$-second row. Amplitude distributions of nonlinear ring solitons for $V_{1} / V_{2}=0.96$ and uniform lattice are depicted in the third row.

of energy is distributed among neighboring sites making an antisymmetric pattern around the diagonal through the ring center, as depicted in the third row of amplitude distribution plots in Fig. 6 when $V_{1} / V_{2}=0.96$. Note that, in contrast to the family A (and B) modes, this solution family has its largest amplitude on the two ring sites connected with the smaller coupling constant $V_{1}$ [compactness with respect to the strongly bonded trimer sites in Fig. 6(b) is smaller than 0.5 ], and thus it cannot be continued into a single trimer mode for decreasing $V_{1}$. However, these solutions are shown to be unstable in the whole range of existence and detailed analysis of their behavior is beyond the scope of this paper.

The stability of the families of localized modes $A$ and $B$ is considered by applying both the LSA and dynamical simulations. Figure 7 represents the corresponding stability diagram for $A$ family of solutions when $V_{2}$ is set to 1 and $V_{1} \leqslant 1$. The area of stable propagation regime is depicted with light blue (gray) color. In the rest of the existence range, solutions exhibit oscillatory instability during the propagation through the lattice. An example of the instability scenario for the family $A$ is illustrated in Fig. 8. The very weak instabilities, signalled by LSA for small $\beta$, are possibly due to the finite-size effects and decrease with increasing system sizes, as seen in Fig. 8(b). Similar scenarios are well known for other situations with resonances between extended eigenmodes belonging to continuous bands, see, e.g., Refs. $[45,48]$ and references therein. We confirmed by direct simulations that these have no notable effects on the core part of the localized modes, and therefore the corresponding regimes are identified as stable in Fig. 7. On the other hand, the true instability (not vanishing for larger systems) appearing for larger amplitude $[\beta \approx 0.3$ in Fig. 8(a)] can be associated to a resonance between an internal mode of the localized solution and extended eigenmodes and results in increasing amplitude oscillations and radiation from the localized solution center.

While a range of stable propagation of family $A$ solutions is found to exist, solutions of family $B$ exhibit unstable behavior in the whole range of existence. In the case of the family $A$ solutions, the widest range for $\beta$ of stable propagation occurs for $V_{1} / V_{2}=0.5$, while it narrows as we approach to the limits

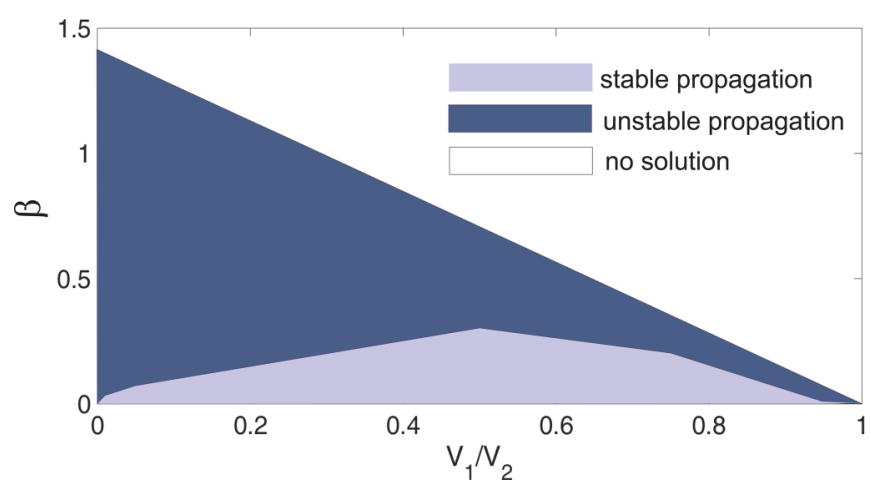

FIG. 7. Stability diagram for the solution of $A$ family of the type I dimerized Lieb lattice for $V_{1}<1$ and $V_{2}=1$. 

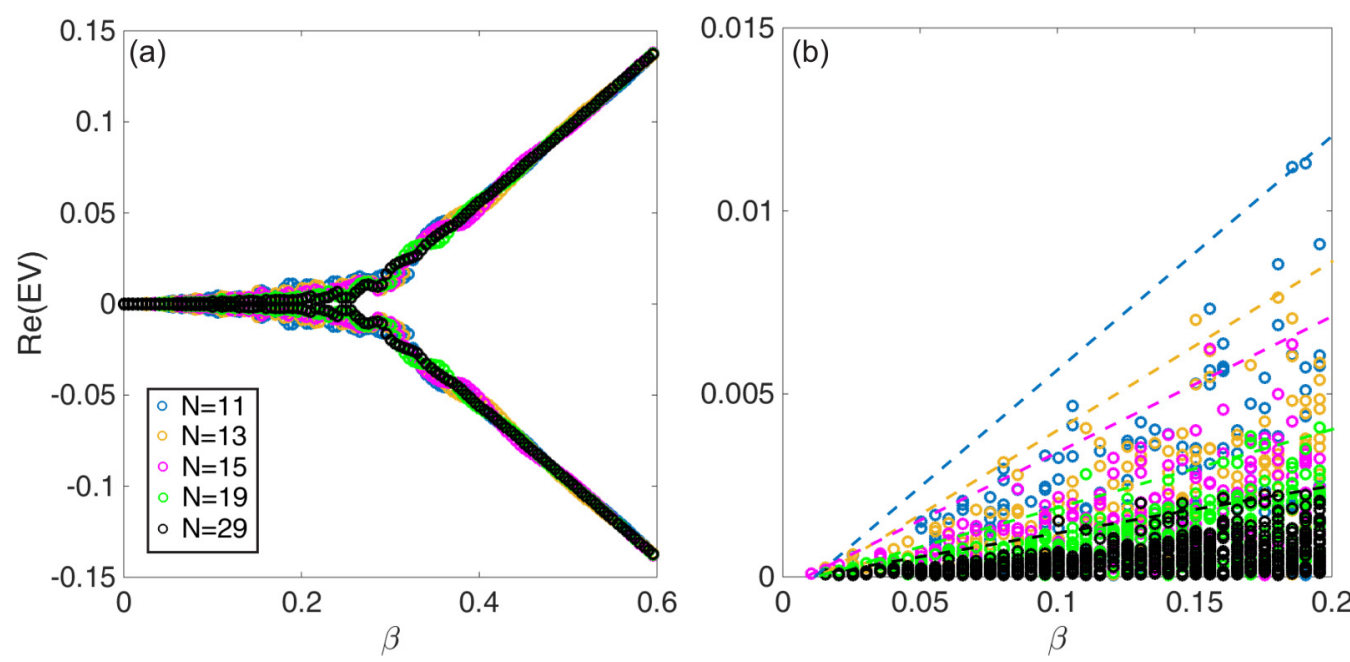

FIG. 8. (a) Maximum values of real parts of stability EVs vs $\beta$ for the solution of family $A$ in the type I dimerization with $V_{1}=0.5$ and $V_{2}=1$, and different system sizes with the number of unit cells denoted by $N \times N$, where $N$ varies between 11 and 29 (periodic boundary conditions). (b) Magnification of (a) for smaller $\beta$. The dashed lines depict growth trend of EVs.

which correspond to the cases of uncoupled antisymmetric trimer modes $\left(V_{1} / V_{2} \rightarrow 0\right)$ and uniform lattice $\left(V_{1} / V_{2} \rightarrow 1\right)$. In the anticontinuous limit the trimer solutions (Fig. 6) are themselves unstable for $0<\beta<2 \sqrt{2} V_{2}$ [49]. On the other hand, as we approach to the limit of uniform lattice, nonlinear localized modes lose their stability due to the proximity of dispersive bands.

If a finite perturbation of $1 \%$ of amplitude on the particular site is added to a stationary mode in the stable regime of Fig. 7, one may observe very long-lived "breathing" oscillations as illustrated in Fig. 9(b). These are explained by the existence of low-frequency internal modes in the linearized spectrum, see Figs. 9(a) and 9(d). When the internal mode frequency approaches the continuous band $(\beta \approx 0.2)$, its second harmonic lies inside the band and second order resonances take place. On the other hand, for smaller $\beta$ only higher frequency harmonics with corresponding small peaks as shown in Fig. 9(d) enter the continuous spectrum, and thus in this regime the radiative
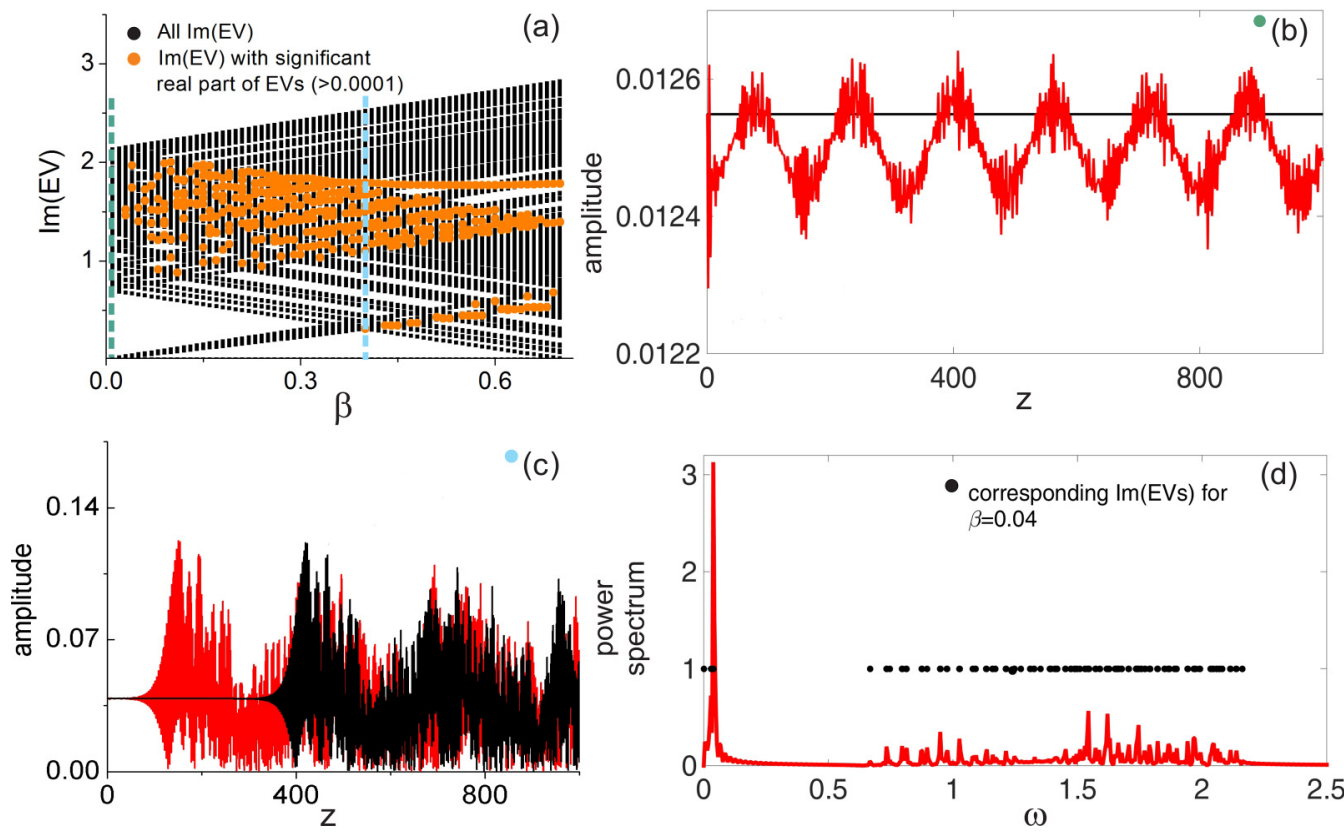

FIG. 9. Dynamics of gap solitons (family $A$ ) for $V_{1}=0.5$ and $V_{2}=1$. (a) The plot of the upper part of full spectrum of stability EVs [positive $\operatorname{Im}(\mathrm{EV})$ ] vs $\beta$. Examples of oscillating dynamics of gap solitons for (b) $\beta=0.04$ (no apparent decay of oscillations) and (c) $\beta=0.42$ (unstable regime). Red (gray) lines in plots (b) and (c) depict profiles of amplitude on $a$ site $[(m, n)=(12,13)$ in Fig. 5(b)] with added perturbation, while the black one stands for the case when the mode evolves without presence of perturbations (only affected by numerical round-off errors). Green (left) and blue (right) vertical dashed lines in (a) correspond to the modes depicted in (b) and (c), respectively. Plot (d) depicts spectrum of amplitude presented in (b) with corresponding positive $\operatorname{Im}(\mathrm{EVs})$. 

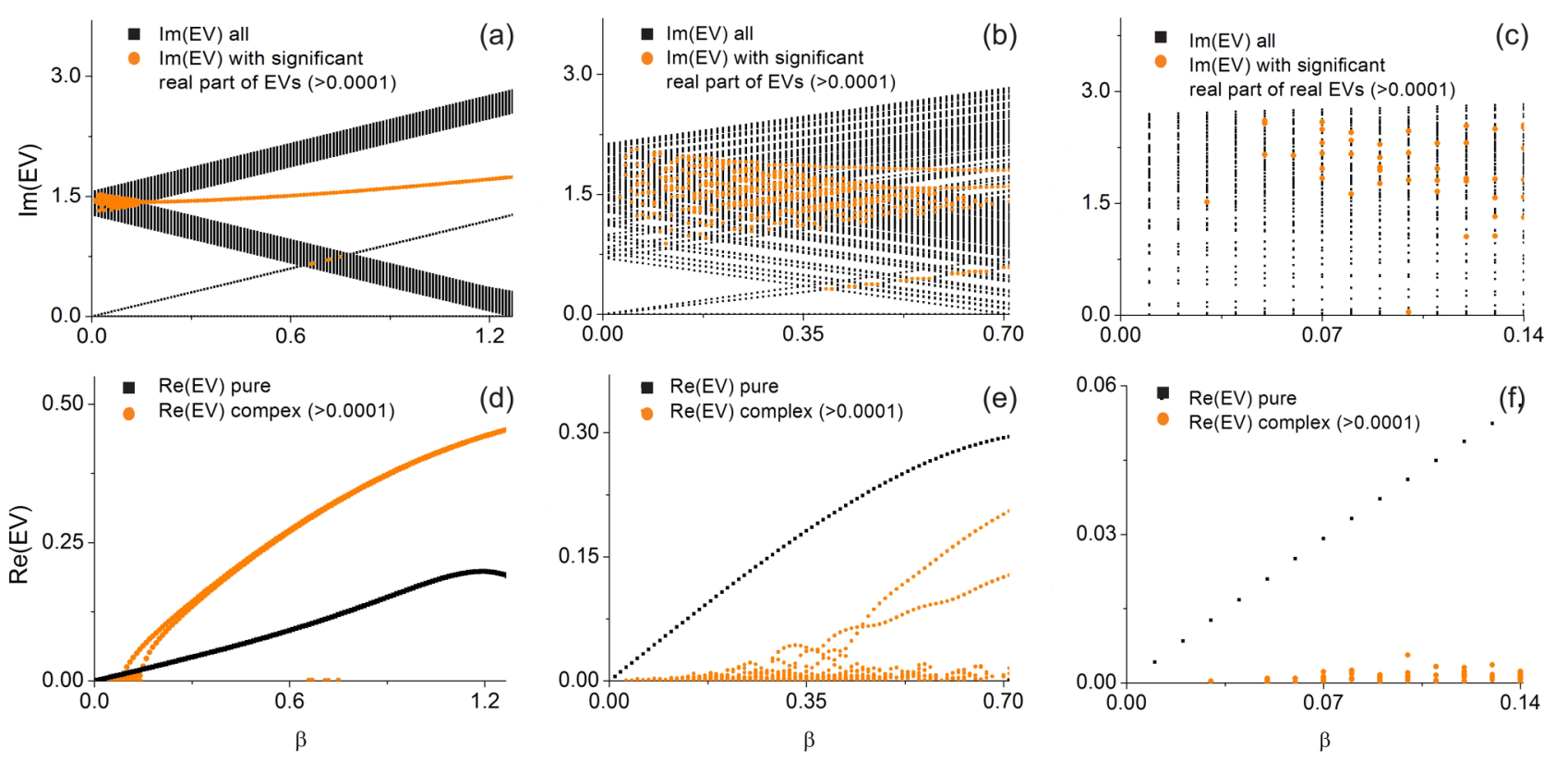

FIG. 10. Upper part of full spectrum of stability EVs [positive $\operatorname{Im}(\mathrm{EVs})$ ] vs $\beta$ for coupling ratios (a) $V_{1} / V_{2}=0.1$, (b) $V_{1} / V_{2}=0.5$, and (c) $V_{1} / V_{2}=0.9$, and corresponding positive $\operatorname{Re}(\mathrm{EVs})$ vs $\beta$ in (d), (e), and (f). Size of the system is $7 \times 7$.

decay of the oscillations becomes extremely slow [Fig. 9(b)] and probably unnoticeable in any experimentally feasible setup. The low-frequency internal modes are seen in Fig. 9(a) to become resonant with the continuous spectrum for $\beta \approx 0.4$, yielding instabilities for larger $\beta$. However, as seen in Fig. 8, the mode becomes unstable already for smaller values of $\beta$. By comparison, we may conclude that the very weak size-dependent instabilities for small $\beta$ in Fig. 8 correspond to overlapping bands in Fig. 9(a), while the surviving instability for $\beta \gtrsim 0.3$ corresponds to a localized eigenmode which is seen to bifurcate from the upper edge of the band with negative slope in Fig. 9(a), resonating with the band with positive slope. An example of dynamics in a regime with several unstable EVs is shown in Fig. 9(c).

Both solution families have very small, almost insignificant power thresholds necessary for their existence (minimal $P$ for every ratio $V_{1} / V_{2}$ is in the order of $10^{-3}$ or smaller). However, while the $A$ family can be found for all ratios, the existence range of $B$ family of solutions is narrower and extends up to $V_{1} / V_{2}<0.65$.

To summarize this part on the type I dimerization, we can conclude that dynamically stable breathing localized modes can be found close to the flat band. This is an important result because it refers to a significance of the dimerization. In other words, the additional periodicity in the lattice pattern opens gaps in the energy spectrum which offers possibility for formation of nonlinear localized modes.

\section{B. Type II dimerization: Nonlinear compact modes}

On the other hand, analysis analogous to that of Eq. (8) shows that the arrangement of couplings between sites proposed in Fig. 1(c) also provides the existence of nonlinear stationary compact modes (ring modes) in the dimerized Lieb lattice of type II. Namely, for such a dimerized configuration, all sites of the linear ring mode have amplitudes of equal absolute values as shown in Sec. II A (Fig. 4), and thus every site gets the same nonlinear frequency shift $\beta=\gamma|A|^{2}$ from the on-site nonlinearity. Consequently, there is a continuation from the linear compact modes into a nonlinear branch of compact homogeneous four-site ring solutions with respect to the nonlinearity parameter, with power given by $P=4 \beta / \gamma$. Therefore, we can conclude that by a particular arrangement of the lattice geometry, where an enlarged unit cell of 12 sites is needed in order to sustain rings with both diagonal and antidiagonal symmetry axes in the presence of dimerization, nonlinear ring modes can be managed. In the following, we will consider properties of such topology.

Nonlinear ring modes in the dimerized lattice of type II are found to exist in the minigap, as well as in the semi-infinite gap above the linear spectrum, Fig. 3(c). However, the LSA generally indicates the instability of these nonlinear ring modes inside the minigap as shown in Fig. 10.

Several features of these instabilities can be understood analytically from certain limiting cases. First, in the anticontinuous limit $\left(V_{1} / V_{2} \rightarrow 0\right)$, the lattice can be viewed as a system of weakly coupled trimers and the nonlinear ring solution reduces to a superposition of two identical single trimer solutions positioned within the ring block (cf. Fig. 4). As shown in Ref. [49], these solutions are unstable. From the exact expression for the stability EVs $\lambda$ derived in Ref. [49], we obtain for small $\beta \ll V_{2}$ (i.e., weak nonlinearity) $\lambda= \pm \mathrm{i} \sqrt{2} V_{2} \pm \beta / 2+O\left(\beta^{2}\right)$. These instabilities will persist also for small but nonzero $V_{1} \ll \beta$, resulting in the complex instabilities seen in Figs. 10(a) and 10(d). The corresponding eigenvectors are symmetric with respect to the diagonal, and thus break the spatial antisymmetry of the ring mode, and correspond to perturbations which populate the originally empty middle site in each trimer. In addition, we note in Fig. 10 a purely real EV which is not present in the anticontinuous limit, but which grows and becomes the dominating instability mechanism when $V_{1} / V_{2}$ increases [Figs. 10(e) and 10(f)]. This 

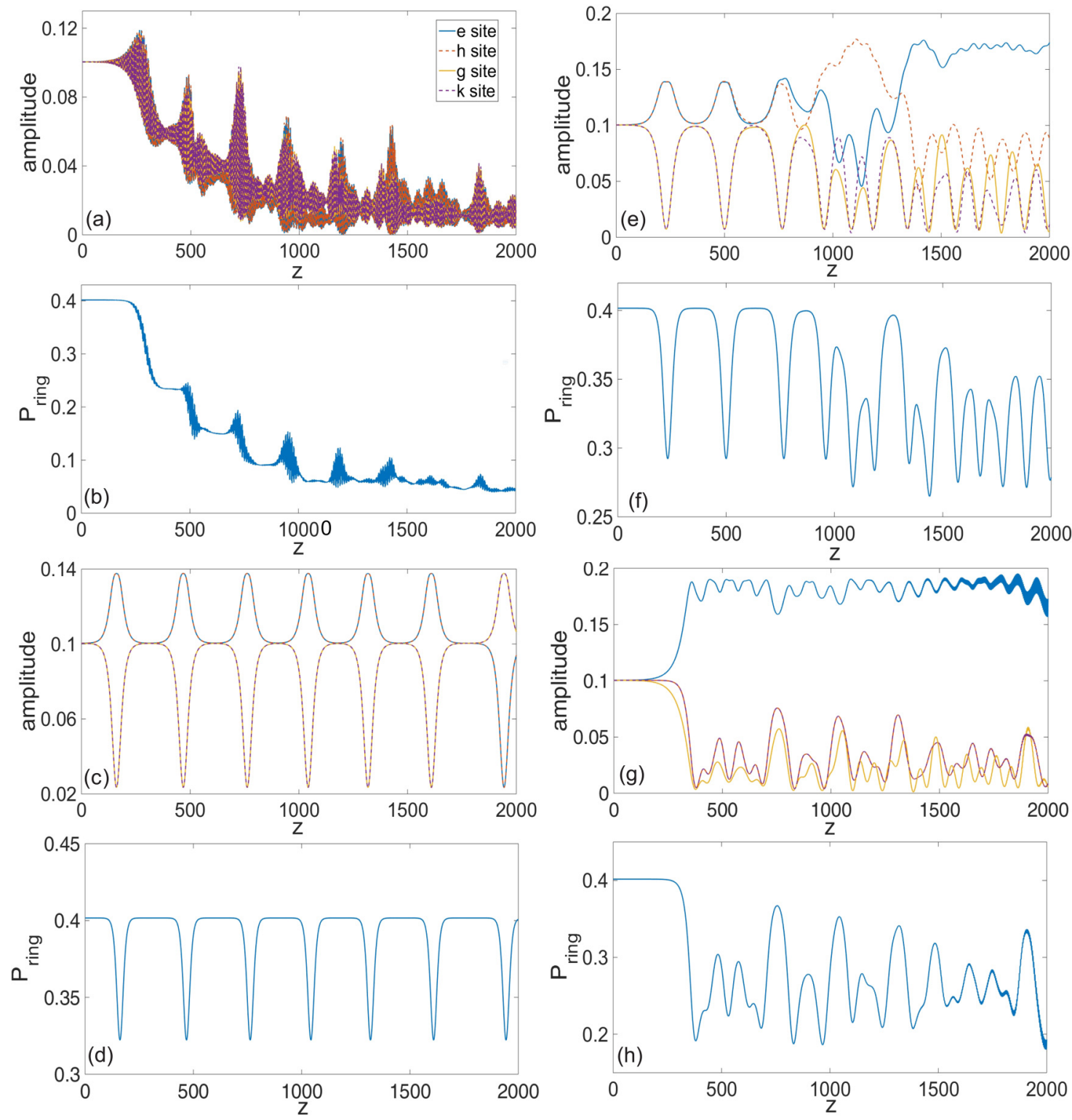

FIG. 11. Moduli of amplitudes on ring sites with the corresponding power of a perturbed nonlinear ring mode, characterized by $\beta=0.1$, in a dimerized Lieb lattice of type II with (a), (b) $V_{1} / V_{2}=0.1$, (c), (d) $V_{1} / V_{2}=0.5$, (e), (f) $V_{1} / V_{2}=0.9$, and (g), (h) $V_{1} / V_{2}=1$. In all cases $V_{2}$ is set to 1 .

EV belongs to a different symmetry class, with eigenvector antisymmetric with respect to the diagonal but symmetric with respect to the antidiagonal, implying that the middle sites in the strongly bonded trimers remain unexcited, while exciting instead the weakly bonded sites connecting two trimers. By assuming $\lambda, \beta, V_{1} \ll V_{2}$, one may obtain from a straightforward perturbation calculation for this symmetry class that, for the unstable $\mathrm{EV}, \lambda \approx \pm \sqrt{2} \beta V_{1} / V_{2}$ to lowest order. Likewise, we obtain also from this symmetry class a stable eigenmode with $\lambda \approx \pm \mathrm{i} \beta$, which as seen in Figs. 10(a) and 10 (b) generates additional weak oscillatory instabilities as it collides with the band spectrum for larger $\beta$.

Direct numerical simulations show that the dynamics of nonlinear ring modes and evolution of instabilities indeed depend strongly on the ratio of coupling constants. In all cases a certain amount of energy exchange between the background and the lattice sites at which the ring is localized has been observed. Figure 11 depicts dynamics of nonlinear ring modes through moduli of amplitudes on ring sites $(e, h, g$, and $k$ sites) as well as power change on corresponding ring sites for different coupling ratios. Here, random perturbation has been added only on sites occupied with a nonlinear ring. The amplitude of perturbation equals $1 \%$ of the value of the amplitude at the particular site. Explicit examples of resulting (moduli of) amplitude distributions for two cases with qualitatively different dynamics are shown in Fig. 12.

In the range of coupling ratios when $V_{1} \ll V_{2}$, nonlinear ring modes tend to delocalize whereby most of the energy spreads across the lattice. An example of propagation of a perturbed nonlinear ring mode $(\beta=0.1)$ through a lattice with coupling ratio $V_{1} / V_{2}=0.1$ is depicted in Fig. 11(a). Decrease in power [Fig. 11(b)] is evident implying radiation of energy 

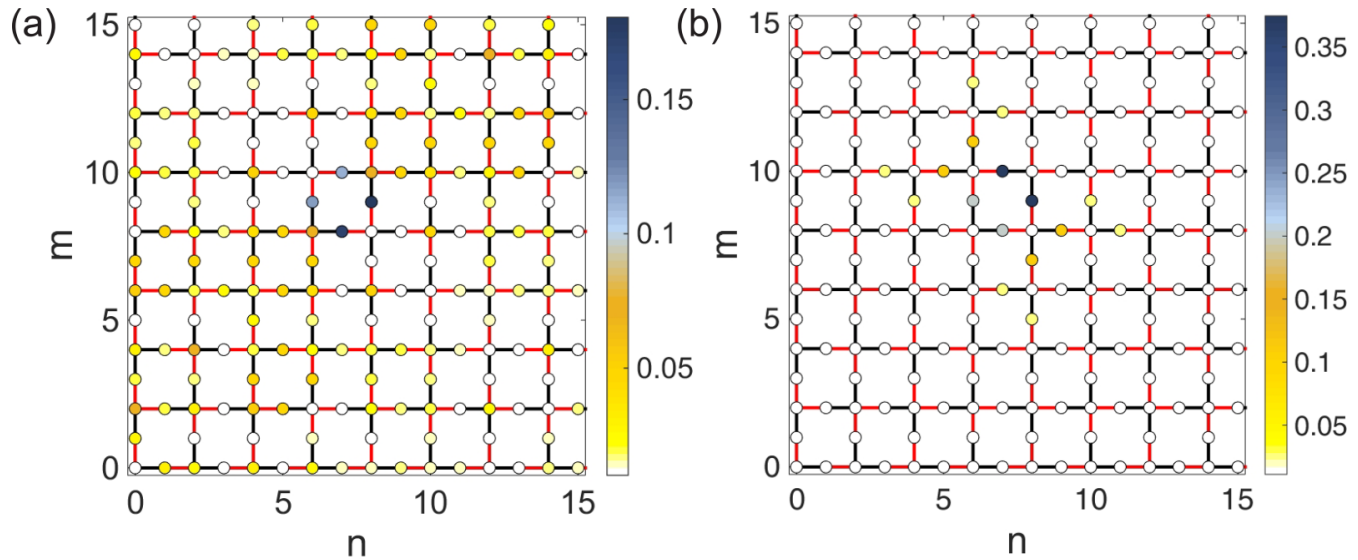

FIG. 12. Moduli of amplitude distributions of perturbed ring modes at $z=1045$ in a dimerized Lieb lattice of type II with (a) $V_{1} / V_{2}=0.1$ and (b) $V_{1} / V_{2}=0.5$.

from the "ring sites." Figure 12(a) shows the corresponding amplitude distribution at $z=1045$. Note that the pattern of amplitude moduli is very close to symmetric with respect to the antidiagonal through the initial ring mode, which indicates that the amplitude pattern itself essentially keeps its antidiagonal antisymmetry, while the (anti)symmetry around the diagonal is clearly broken. This is a typical outcome in the regime dominated by the complex instability in Figs. 10(a) and 10(d).

With decreasing dimerization strength (increasing $V_{1} / V_{2}$ ), the dynamics of the solution goes into a regime with a longlived regular exchange of energy between ring and neighboring sites as shown in Fig. 11(c), whereby power on "ring sites" can drop to around 70\% of the total mode's power [Fig. 11(d)]. As can be seen, energy exchange also occurs within the ring formation itself: between $e$ and $h$, and $g$ and $k$ sites. The corresponding amplitude distribution at $z=1045$ is shown in Fig. 12(b). Although the power on ring sites has minimum value at this propagation length $z=1045$ [see Fig. 11(d)], most of the energy stays localized on and close to the ring sites preserving the ring formation of the mode as shown in Fig. 12(b). As also can be seen, in contrast to the case in Fig. 12(a), the mode remains very close to (anti)symmetric around the central diagonal, while the (anti)symmetry is clearly broken around the antidiagonal. This is the typical feature of the regime dominated by the real instability in Fig. 10(e).

As we approach the case of the uniform lattice the situation changes. After a certain propagation length $[z \sim 1300$ for the case with $V_{1} / V_{2}=0.9$ illustrated in Figs. 11(e) and 11(f)], light tends to localize more within one site of the ring [ $e$ site in Fig. 11(e)] weakly interacting with closest neighbors. Thus, even though the transient dynamics is still derived from the real eigenmode in Fig. 10(f) with preserved diagonal antisymmetry, further instabilities will finally also break this symmetry. In the case of uniform lattice, no significant change appears prior to $z \sim 300$ when instability slowly develops and light tends to stay maximally localized only on one site of the ring [ $e$ site in Fig. $11(\mathrm{~g})]$ radiating most of the energy across the lattice.

Generally, the energy exchange rate also grows for modes with $\beta$ taking values away from the flat band position for fixed dimerization strength $V_{1} / V_{2}$. In order to check regularity of energy exchange among the sites of nonlinear ring modes, we took the propagation length in our simulations to be $z=500$. Relying on experimental results published in Ref. [31], in which dimensions of waveguides and separations between them are several microns, and presuming that the coupling constants are dominantly dependent on these quantities, the estimated length of $z=500$ in arbitrary units would correspond to a sample length of $50 \mathrm{~cm}$. This can be considered as a long propagation limit. Based on this, we can state that the main excitation stays close to the ring and that nonlinear ring modes are practically preserved for such experimental setups if the exchange of the energy between ring and neighboring sites during the propagation length $(z=500)$ is regular as shown in Fig. 11(c) and if the power outside the ring sites doesn't exceed more than $1 / 3$ of the total mode's power. If these conditions are satisfied we assume that the nonlinear ring formation has been preserved in relevant experiments.

According to the performed dynamical simulations appropriate "power localization on ring" diagram has been obtained as plotted in Fig. 13. The plot shows two different regimes within the existence range of nonlinear ring modes, in which the observed ring mode is viewed as preserved [light blue

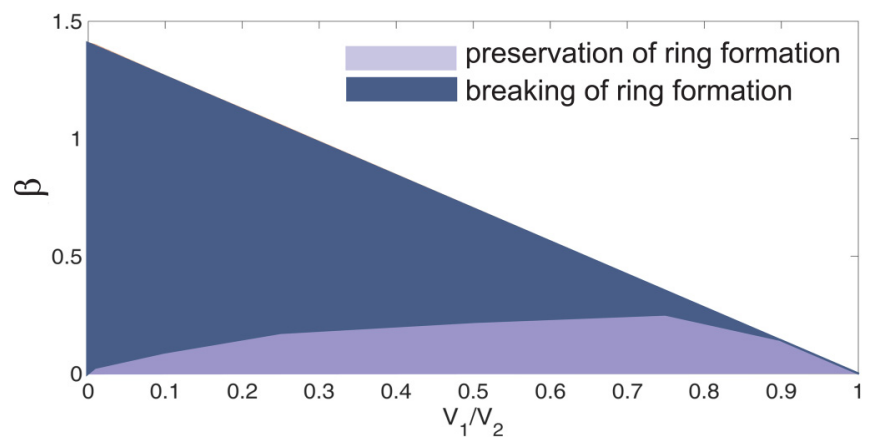

FIG. 13. Parameter diagram depicting regimes of preservation [light blue (gray) color] and breaking [deep blue (dark gray) color] of four-site nonlinear ring formation when $0<V_{1}<1$ and $V_{2}=1$, of the type II dimerized Lieb lattice. Regimes are defined upon two criteria: regular exchange of energy along the propagation length $z=500$ and power preservation of at least $2 / 3$ of total modes' power on four main sites. Line between deep blue (dark gray) and white region represents upper border of minigap. 
(gray) color in Fig. 13] if the above criteria are fulfilled. As can be seen, this region gets widest for coupling ratio $V_{1} / V_{2}=0.75$. Approaching to the anticontinuous limit, the nonlinear ring mode can be considered as a superposition of two uncoupled unstable trimer modes which leads to delocalization of the nonlinear ring mode. In the limit of uniform Lieb lattice we observe light localization dominantly on one site of the ring. It is interesting to note that the regime characterized as experimentally preserved in Fig. 13 largely coincides with the regime where the instability scenario is strongly dominated by the real EV preserving the diagonal antisymmetry in Figs. 10(d)-10(f).

Finally, it is worth noting that the same procedure has been done when random perturbation is added on all sites of the lattice and equals $1 \%$ of the amplitude value on site $e$. Similar behavior of amplitudes' dynamics has been observed whereas delocalization of nonlinear ring modes occurs later for the case of random perturbations added only on ring sites. The reason for this is absence of noise and smaller coupling between zero amplitude neighboring sites.

\section{CONCLUSIONS}

In this paper we have considered the mutual effects of the nonlinearity and dimerization, characterized by the opening of gaps in the EV spectra, on the different localized mode generation and propagation in $2 \mathrm{D}$ Lieb lattices. We have considered two explicit dimerization patterns with different symmetry properties and unit cell sizes. Nonlinear compact localized modes, emerging inside the newly opened minigaps from the linear flat band ring solutions, were shown to form only in the dimerized lattice having both diagonal and antidiagonal symmetry axes ("type II"). For the dimerization with only diagonal reflection symmetry ("type I"), we have instead shown the possibility to generate nonlinear stationary and "breathing" localized modes inside the minigaps, which are dynamically stable in the vicinity of the flat band. While the ring mode in the type II nonlinear dimerized lattices is reminiscent of the linear flat band ring, the localized breathing modes found in the minigaps of the type I lattice are highly localized structures which can carry a large amount of energy within just a few sites. Our findings might open a new pathway in the attempts to realize the most suitable environment and conditions for full control of the light propagation in photonics. Some experimental realizations of electronic Lieb lattices have also been done recently using $\mathrm{CO}$ molecules on $\mathrm{Cu}(111)$ [50]. The proposed system may be a good candidate for our predictions, since it allows one to tune parameters that cannot be easily varied in a real solidstate material. Moreover, with the dint of femtosecond laser inscription technique it is possible to realize various lattice patterns. For instance, in Refs. [51,52] experiments showed that weak coupling was required to obtain nonlinear localized solutions at the surface of femtosecond laser written nonlinear square lattices. Having in mind that in dimerized Lieb lattice soliton solution bifurcates from the linear limit, its geometry can provide conditions for tailoring strength of coupling.

\section{ACKNOWLEDGMENTS}

We are grateful to Rodrigo Vicencio and Bastián Real for discussions on the Lieb lattice and for sharing their results [42] prior to publication. The authors acknowledge support from the Swedish Research Council through the project "Control of light and matter waves propagation and localization in photonic lattices" within the Swedish Research Links programme, 348-2013-6752, and the Ministry of Education, Science and Technological Development of the Republic of Serbia (project III 45010).
[1] Photonic Crystals and Light Localization in the 21st Century, edited by C. M. Soukoulis (Springer, Dordrecht, 2001).

[2] M. Segev, Y. Silberberg, and D. N. Christodoulides, Nat. Photon. 7, 197 (2013).

[3] H. Trompeter, U. Peschel, T. Pertsch, F. Lederer, U. Streppel, D. Michaelis, and A. Bräuer, Opt. Express 11, 3404 (2003); J. M. Lourtioz, H. Benisty, V. Berger, J. M. Gerard, D. Maystre, and A. Tchelnokov, Photonic Crystals: Towards Nanoscale Photonic Devices, 2nd ed. (Springer-Verlag, Berlin, 2008).

[4] V. E. Zakharov and L. A. Ostrovsky, Physica D 238, 540 (2009).

[5] F. Lederer, G. I. Stegeman, D. N. Christodoulides, G. Assanto, M. Segev, and Y. Silberberg, Phys. Rep. 463, 1 (2008).

[6] W. Chen and D. L. Mills, Phys. Rev. Lett. 58, 160 (1987).

[7] A. A. Sukhorukov and Yu. S. Kivshar, Opt. Lett. 27, 2112 (2002).

[8] R. Morandotti, D. Mandelik, Y. Silberberg, J. S. Aitchison, M. Sorel, D. N. Christodoulides, A. A. Sukhorukov, and Yu. S. Kivshar, Opt. Lett. 29, 2890 (2004).

[9] R. A. Vicencio and M. Johansson, Phys. Rev. A 79, 065801 (2009).

[10] A. Kanshu, C. E. Rüter, D. Kip, V. Shandarov, P. P. Beličev, I. Ilić, and M. Stepić, Opt. Lett. 37, 1253 (2012).
[11] J. D. Joannopoulos, P. R. Villeneuve, and S. H. Fan, Nature (London) 386, 143 (1997).

[12] M. C. Rechtsman, J. M. Zeuner, Y. Plotnik, Y. Lumer, D. Podolsky, F. Dreisow, S. Nolte, M. Segev, and A. Szameit, Nature (London) 496, 196 (2013).

[13] M. Hafezi, S. Mittal, J. Fan, A. Migdall, and J. Taylor, Nat. Photon. 7, 1001 (2013).

[14] H. Tasaki, Eur. Phys. J. B 64, 365 (2008).

[15] D. L. Bergman, C. Wu, and L. Balents, Phys. Rev. B 78, 125104 (2008).

[16] L. Zheng, L. Feng, and W. Yong-Shi, Chin. Phys. B 23, 077308 (2014).

[17] T. Neupert, L. Santos, C. Chamon, and C. Mudry, Phys. Rev. Lett. 106, 236804 (2011); E. J. Bergholtz and Z. Liu, Int. J. Phys. B 27, 1330017 (2013).

[18] X. Cao, K. Chen, and D. He, J. Phys.: Condens. Matter 27, 166003 (2015).

[19] J. Vidal, R. Mosseri, and B. Doucot, Phys. Rev. Lett. 81, 5888 (1998).

[20] C. Weeks and M. Franz, Phys. Rev. B 82, 085310 (2010).

[21] G. Gligorić, A. Maluckov, Lj. Hadžievski, S. Flach, and B. A. Malomed, Phys. Rev. B 94, 144302 (2016). 
[22] D. Leykam, S. Flach, O. Bahat-Treidel, and A. S. Desyatnikov, Phys. Rev. B 88, 224203 (2013).

[23] J. D. Bodyfelt, D. Leykam, C. Danieli, X. Yu, and S. Flach, Phys. Rev. Lett. 113, 236403 (2014).

[24] M. I. Molina, Phys. Lett. A 376, 3458 (2012).

[25] P. P. Beličev, G. Gligorić, A. Radosavljević, A. Maluckov, M. Stepić, R. A. Vicencio, and M. Johansson, Phys. Rev. E 92, 052916 (2015).

[26] M. Boguslawski, P. Rose, and C. Denz, Appl. Phys. Lett. 98, 061111 (2011).

[27] R. A. Vicencio and C. Mejía-Cortés, J. Opt. 16, 015706 (2014).

[28] K. J. H. Law, A. Saxena, P. G. Kevrekidis, and A. R. Bishop, Phys. Rev. A 79, 053818 (2009).

[29] R. A. Vicencio and M. Johansson, Phys. Rev. A 87, 061803(R) (2013).

[30] D. Leykam, O. Bahat-Treidel, and A. S. Desyatnikov, Phys. Rev. A 86, 031805(R) (2012).

[31] R. A. Vicencio, C. Cantillano, L. Morales-Inostroza, B. Real, C. Mejía-Cortés, S. Weimann, A. Szameit, and M. I. Molina, Phys. Rev. Lett. 114, 245503 (2015); D. Guzmán-Silva, C. Mejía-Cortés, M. A. Bandres, M. C. Rechtsman, S. Weimann, S. Nolte, M. Segev, A. Szameit, and R. A. Vicencio, New J. Phys. 16, 063061 (2014).

[32] S. Mukherjee, A. Spracklen, D. Choudhury, N. Goldman, P. Öhberg, E. Andersson, and R. R. Thomson, Phys. Rev. Lett. 114, 245504 (2015).

[33] S. Xia, Y. Hu, D. Song, Y. Zong, L. Tang, and Z. Chen, Opt. Lett. 41, 1435 (2016).

[34] F. Diebel, D. Leykam, S. Kroesen, C. Denz, and A. S. Desyatnikov, in Advanced Photonics, OSA Technical Digest (online) (Optical Society of America, Washington, DC, 2014), paper NW3A.1.
[35] K. Noda, K. Inaba, and M. Yamashita, Phys. Rev. A 90, 043624 (2014).

[36] C. Poli, H. Schomerus, M. Bellec, U. Kuhl, and F. Mortessagne, 2D Mater. 4, 025008 (2017).

[37] A. Julku, S. Peotta, T. I. Vanhala, D.-H. Kim, and P. Törmä, Phys. Rev. Lett. 117, 045303 (2016).

[38] L. Ge, Phys. Rev. A 92, 052103 (2015).

[39] W. Beugeling, J. C. Everts, and C. Morais Smith, Phys. Rev. B 86, 195129 (2012).

[40] G. Palumbo and K. Meichanetzidis, Phys. Rev. B 92, 235106 (2015).

[41] B. Jaworowski, A. Manolescu, and P. Potasz, Phys. Rev. B 92, 245119 (2015).

[42] B. Real and R. A. Vicencio (private communication, 2016).

[43] R. Shen, L. B. Shao, B. Wang, and D. Y. Xing, Phys. Rev. B 81, 041410(R) (2010).

[44] J. Carr and J. C. Eilbeck, Phys. Lett. 109A, 201 (1985).

[45] S. Flach and A. V. Gorbach, Phys. Rep. 467, 1 (2008).

[46] P. P. Beličev, G. Gligorić, A. Maluckov, and M. Stepić, Europhys. Lett. 104, 14006 (2013).

[47] G. Gligorić, A. Maluckov, Lj. Hadžievski, and B. A. Malomed, Phys. Rev. A 78, 063615 (2008).

[48] A. V. Gorbach and M. Johansson, Eur. Phys. J. D 29, 77 (2004).

[49] C. Schmidt-Hattenberger, R. Muschall, U. Trutschel, and F. Lederer, Opt. Quantum Electron. 24, 691 (1992).

[50] M. R. Slot, T. S. Gardenier, P. H. Jacobse, G. C. P. van Miert, S. N. Kempkes, S. J. M. Zevenhuizen, C. M. Smith, D. Vanmaekelbergh, and I. Swart, Nat. Phys. 13, 672 (2017).

[51] A. Szameit, J. Burghoff, T. Pertsch, S. Nolte, A. Tünnermann, and F. Lederer, Opt. Express 14, 6055 (2006).

[52] A. Szameit, Y. V. Kartashov, F. Dreisow, T. Pertsch, S. Nolte, A. Tünnermann, and L. Torner, Phys. Rev. Lett. 98, 173903 (2007). 